\begin{tabular}{|c|l|}
\hline Title & Action minimization for an A llen-Cahn equation with an unequal double well potential \\
\hline Author(s) & Nagase, Yuko \\
\hline Citation & $\begin{array}{l}\text { Manuscripta Mathematica, 1371-2), 81-106 } \\
\text { https://doi.org/10.1007/300229-011-0458-5 }\end{array}$ \\
\hline Issue Date & 2012-01 \\
\hline Doc URL & http://hdl.handle.net/2115/51013 \\
\hline Rights & The original publication is available at www.springerlink.com \\
\hline Type & article (author version) \\
\hline File Information & MM137-1-2_81-106.pdf \\
\hline
\end{tabular}

Instructions for use 


\title{
Action minimization for an Allen-Cahn equation with an unequal double-well potential
}

\author{
Yuko Nagase \\ Department of Mathematics, Faculty of science, Hokkaido university, \\ Sapporo, 060-0810, JAPAN \\ nagase@math.sci.hokudai.ac.jp
}

\begin{abstract}
We consider the minimization problem of the Allen-Cahn action functional with an unequal double-well potential. For the stochastic Allen-Cahn equation switching from one stable state to the other rarely occurs. The probability of switching is determined by the minimum of the action functional. We give an explicit description of the minimum and its optimal path in one-dimension.
\end{abstract}

Mathematics Subject Classification (2000) : 49Q20, 35B25, 82B26, 82B24

Keyword : variational problem, stochastic Allen-Cahn equation, unequal double-well potential, action functional

\section{Introduction}

In this paper we consider the minimization problem of the scaled Allen-Cahn action functional with an unequal double-well potential in one-dimension, that is, for $\varepsilon>0$ and $u:[0, L] \times[0, T] \rightarrow \mathbb{R}$

$$
S_{\varepsilon}^{\theta}(u):=\frac{1}{4} \int_{0}^{T} \int_{0}^{L}\left\{\sqrt{\varepsilon} u_{t}+\frac{1}{\sqrt{\varepsilon}}\left(-\varepsilon u_{x x}+\frac{W_{\varepsilon}^{\prime}(u)}{\varepsilon}\right)\right\}^{2} d x d t,
$$

where $W_{\varepsilon}: \mathbb{R} \rightarrow \mathbb{R}$ is a double-well potential whose well-depths differ by order $\varepsilon$, namely

$$
W_{\varepsilon}(s)=W(s)+\theta \varepsilon s
$$

where $W$ is an equal double-well potential which has minima at \pm 1 and $\theta>0$ is a fixed constant. The potential $W_{\varepsilon}$ has two minima which deviate from \pm 1 only slightly when $\varepsilon$ is small. We denote by $\alpha_{\varepsilon}$ and $\beta_{\varepsilon}$ the two minimum points close to -1 and +1 respectively. 


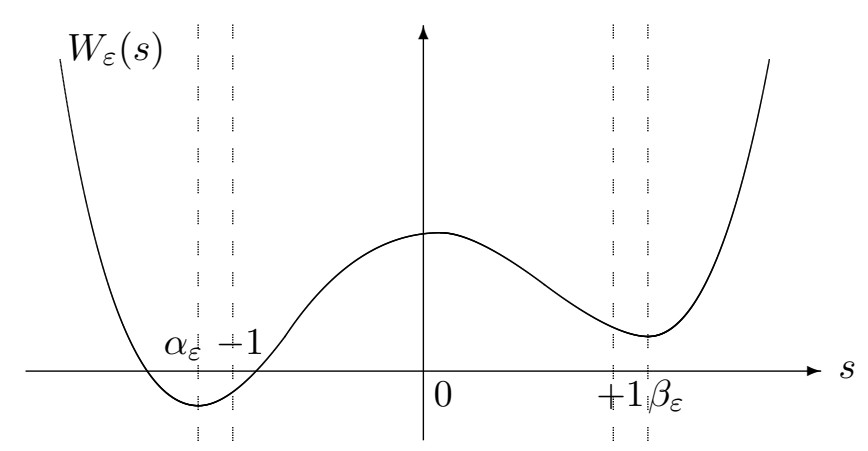

This action functional originally comes from the stochastic Allen-Cahn equation. Switching between two deterministically stable states rarely occurs for the Allen-Cahn equation driven by white noise (cf.(2.1)). The probability of this rare event is estimated by the Wentzell-Freidlin theory of large deviations through the minimization problem of the action functional.

A mathematical analysis of the equal well case, namely $\theta=0$, is found in [8], [9], [12] and [17]. The $\Gamma$-convergence of the action functionals in one-dimension has been proved in [9] and [17] and in higher dimensions $(n=2,3)$ in [12].

In particular, in one-dimension, if we assume the single multiplicity of the interface measure, which is a delta-measure on the interfaces between two stable states (for the exact expression of such measure, see Lemma 5.4), the action functionals $S_{\varepsilon}^{0}$ formally converges to

$$
\sum_{k=1}^{M-1}\left\{\frac{c_{0}}{2}|N(k+1)-N(k)|+\frac{c_{0}}{4} \int_{T_{k}}^{T_{k+1}} \sum_{i=1}^{N(k)} \dot{g}_{i}(t)^{2} d t\right\},
$$

where $\left\{T_{k}\right\}_{k=1}^{M}$ is a sequence of times when new interfaces are created, $N(k)$ is the total number of interfaces at time $T_{k}$, the constant $c_{0}:=\int_{-1}^{1} \sqrt{2 W(s)} d s$ and $g_{i}(t)$ is the location of the $i^{t h}$ interface at time $t$.

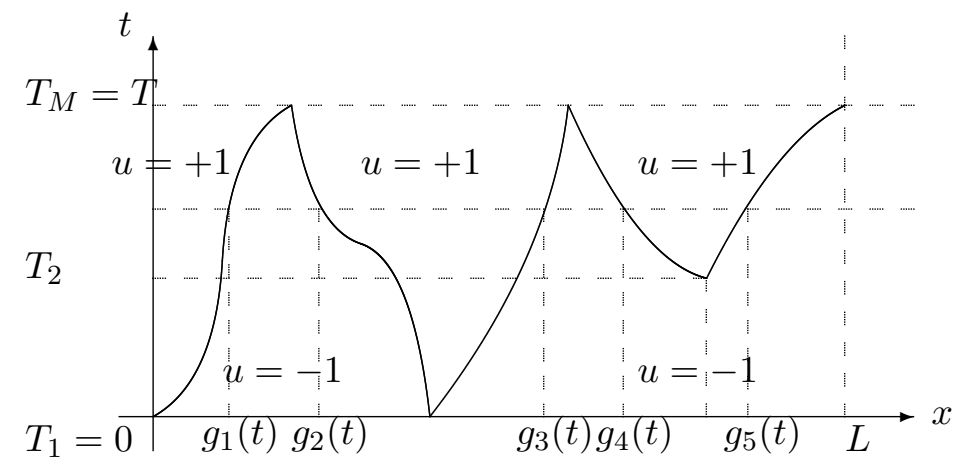


From the limit (1.3) we can interpret the action functional as the sum of "the nucleation cost," that is, the cost of creating new interfaces which cause jumps in energy levels, which is the first term of (1.3), and a "propagation cost" which depends on the speed of the moving interface, which is the second term of (1.3).

We remark that, of course, we have to be very careful about the multiplicity of the interface measure especially in the weak formulation used geometric measure theory. Actually in [17] they remove the assumption of the single multiplicity by defining certain class of measures and admissible functions. In this paper, in order to focus on observing the effect of the difference of the well-depths, we assume the single multiplicity of the interface measure (see remark 5.5).

Our problem is what happens when the depth of the wells differs by order $\varepsilon$. We can expect that the velocity of the moving interfaces will be affected by the difference of the well-depths and this effect will appear in the propagation cost. In order to show such a phenomenon, we analyze the switching problem from the deeper stable state under the Dirichlet boundary condition by means of studying the limit of the minimum of the action functional $S_{\varepsilon}^{\theta}$ among all functions $u \in C^{\infty}([0, L] \times[0, T] ; \mathbb{R})$ with $u(x, 0)=\alpha_{\varepsilon}$ and $u(x, T)=\beta_{\varepsilon}$, as $\varepsilon$ tends to 0 . As our main result we explicitly describe the limit value of the minimun of the action functional and its optimal switching path.

The analysis of interfaces is deeply related to the free energy in the Van der WaalsCahn-Hillard theory of phase transitions, defined by

$$
E_{\varepsilon}(u)=\int_{0}^{L} \frac{\varepsilon\left|u_{x}\right|^{2}}{2}+\frac{W(u)}{\varepsilon} d x .
$$

In a stationary problem the basic result by Modica \& Mortola and Sternberg shows that for general dimension $n$ the energy $\Gamma$-converges to the constant $c_{0}$ times the $(n-1)$-dimensional Hausdorff measure of the interface between $\{u=+1\}$ and $\{u=-1\}$ as $\varepsilon$ tends to 0 , proved in [14] and [11]. In one-dimension, it implies the number of the jumps between $\{u=1\}$ and $\{u=-1\}$. For our potential $W_{\varepsilon}$ we also define an energy functional $E_{\varepsilon}^{\theta}(u)$ as

$$
E_{\varepsilon}^{\theta}(u):=\int_{0}^{L} \frac{\varepsilon\left|u_{x}\right|^{2}}{2}+\frac{W_{\varepsilon}(u)}{\varepsilon} d x=E_{\varepsilon}(u)+\int_{0}^{L} \theta u d x .
$$

For this energy we cannot ignore the contribution of the difference of the well-depths in the limit. If we fix a time $t$, the energy $E_{\varepsilon}^{\theta}$ should converge to which is the same $\Gamma$-limit as $E_{\varepsilon}$ added to $\theta$ times the difference of the volumes of the regions $\{u=1\}$ and $\{u=-1\}$. We discuss these facts in Section 5 .

The action functional $S_{\varepsilon}^{\theta}$ can be estimated by the difference of the energy $E_{\varepsilon}^{\theta}$ at two 
different times, that is, for any $0 \leq t_{1} \leq t_{2}$, we have

$$
\begin{aligned}
& \left.S_{\varepsilon}^{\theta}(u)\right|_{t=t_{1}} ^{t_{2}} \\
& =\frac{1}{4} \int_{t_{1}}^{t_{2}} \int_{0}^{L}\left\{\sqrt{\varepsilon} u_{t}-\frac{1}{\sqrt{\varepsilon}}\left(-\varepsilon u_{x x}+\frac{W_{\varepsilon}^{\prime}(u)}{\varepsilon}\right)\right\}^{2}+4 u_{t}\left(-\varepsilon u_{x x}+\frac{W_{\varepsilon}^{\prime}(u)}{\varepsilon}\right) d x d t \\
& =\frac{1}{4} \int_{t_{1}}^{t_{2}} \int_{0}^{L}\left\{\sqrt{\varepsilon} u_{t}-\frac{1}{\sqrt{\varepsilon}}\left(-\varepsilon u_{x x}+\frac{W_{\varepsilon}^{\prime}(u)}{\varepsilon}\right)\right\}^{2} d x d t+\int_{t_{1}}^{t_{2}} \frac{d}{d t} E_{\varepsilon}^{\theta}(u) d t \\
& \geq\left. E_{\varepsilon}^{\theta}(u)\right|_{t=t_{2}}-\left.E_{\varepsilon}^{\theta}(u)\right|_{t=t_{1}} .
\end{aligned}
$$

Thus to study the limit of the energy $E_{\varepsilon}^{\theta}$ it is helpful to understand how the action functionals $S_{\varepsilon}^{\theta}$ behave as $\varepsilon$ tends to 0 .

One of our main ideas is to use a solution of a certain ODE in the transition profile. In order to study the evolution of the interface we consider the optimal profile problem for the energy $E_{\varepsilon}^{\theta}$. While a transition profile is given simply by a hyperbolic tangent in the equal-well case, we expect that it differs from a hypabolic tangent by the terms of order $\varepsilon$. This implies that it is appropriate to use a solution $\phi_{\varepsilon}$ of the ODE,

$$
\lambda_{\varepsilon} \phi_{\varepsilon}^{\prime}=-\phi_{\varepsilon}^{\prime \prime}+W_{\varepsilon}^{\prime}\left(\phi_{\varepsilon}\right) \quad \text { in } \mathbb{R}
$$

under the boundary condition $\phi_{\varepsilon}(-\infty)=\alpha_{\varepsilon}$ and $\phi_{\varepsilon}(+\infty)=\beta_{\varepsilon}$, where $\lambda_{\varepsilon}$ is of order $\varepsilon$, which is studied in [1] and [2]. Near one interface, we have $u(x, t) \approx \phi_{\varepsilon}\left(\frac{1}{\varepsilon}\left(x+\frac{\lambda_{\varepsilon}}{\varepsilon}\right) t\right)$, which implies that the additional speed of the moving interfaces comes from the constant $\frac{\lambda_{\varepsilon}}{\varepsilon}$. We need this discussion for the construction of the upper bound of the action functional in Section 4 .

Organization We state how to relate the action functional to the stochastic Allen-Cahn equation in next section. In Section 3, we state the mathematical setting and our main result. In Section 4, we construct the upper bound. In Section 5, we introduce action measures, prove some required lemmas and show the lower bound.

\section{Connection to stochastic Allen-Cahn equation}

In this section we briefly state the relationship between the stochastic perturbed PDE and the action functional. We consider the following Allen-Cahn equation with a white noise,

$$
\left\{\begin{array}{lr}
u_{t}=u_{x x}-V^{\prime}(u)+\sqrt{2 \gamma} \eta & \text { in }[0, L] \times[0, T] \\
u_{x}(0, t)=u_{x}(L, t)=0 & \text { in }[0, T]
\end{array}\right.
$$

where $\gamma>0$ is a constant, $\eta$ is a space-time white noise and $V: \mathbb{R} \rightarrow \mathbb{R}$ is a double-well potential. For the deterministic case, there are the two stable states $\alpha$ and $\beta$ corresponding to the two minima of the potential $V$. On the other hand, for the stochastic case, the switch 
between the deterministically stable states happens with a small probability. Faris and Jona-Lasinio showed the probablity of this switching is determined by the minimum of the action functional [6]. The action functional associated with (2.1) is defined by

$$
S(u)=\frac{1}{4} \int_{0}^{T} \int_{0}^{L}\left\{u_{t}-u_{x x}+V^{\prime}(u)\right\}^{2} d x d t,
$$

which implies the finite $L^{2}$-norm of the white noise. The probability $P$ of the switching from one stable state $\alpha$ to the $\delta$-neighborhood $B_{\delta}(\beta):=\{y \in \mathbb{R}|| \beta-y \mid<\delta\}$ of the other stable state $\beta$, is given by

$$
\lim _{\gamma \rightarrow 0} \gamma \log P=-\delta S
$$

where $\delta S$ is the minimization of this action functional,

$$
\begin{aligned}
\delta S=\inf \left\{S(u) \mid u \in C^{\infty}([0, L] \times[0, T])\right. & \\
& u_{x}(0, t)=u_{x}(L, t)=0, \\
& \left.u(x, 0)=\alpha, \quad u(x, T) \in B_{\delta}(\beta)\right\} .
\end{aligned}
$$

The probability of switching to the point $\beta$ is obtained by taking limit as $\delta$ tends to 0 in the suitable sense (see [17]).

Therefore our problem is to analyze $\delta S$ and its minimizer. We apply the same terminology to an unequal potential $W_{\varepsilon}$ and consider the suitable scaled problem by rescaling $x \rightarrow \varepsilon x$ and $t \rightarrow \varepsilon^{2} t$.

\section{Assumptions and Main Result}

For $\varepsilon>0$, we consider the action functional defined by

$$
S_{\varepsilon}^{\theta}(u)=\frac{1}{4} \int_{0}^{T} \int_{0}^{L}\left(\sqrt{\varepsilon} u_{t}+\frac{F_{\varepsilon}^{\theta}}{\sqrt{\varepsilon}}\right)^{2} d x d t,
$$

where $W_{\varepsilon}: \mathbb{R} \rightarrow \mathbb{R}$ is a double-well potential whose depths are different given by

$$
W_{\varepsilon}(s):=W(s)+\theta \varepsilon s \quad \text { with } \quad W(s)=\frac{1}{4}\left(1-s^{2}\right)^{2}
$$

for some constant $\theta>0$. We assume there are exactly three points $\alpha_{\varepsilon}<\kappa_{\varepsilon}<\beta_{\varepsilon}$ where $W_{\varepsilon}^{\prime}=0$. We set

$$
F_{\varepsilon}^{\theta}:=-\varepsilon u_{x x}+\frac{W_{\varepsilon}^{\prime}(u)}{\varepsilon}=-\varepsilon u_{x x}+\frac{W^{\prime}(u)}{\varepsilon}+\theta .
$$

We consider the following minimization problem,

$$
\delta S_{\varepsilon}^{\theta}=\inf _{u \in \mathcal{A}_{\varepsilon}} S_{\varepsilon}^{\theta}(u)
$$


where

$$
\begin{gathered}
\mathcal{A}_{\varepsilon}=\left\{u \mid u \in C^{\infty}([0, L] \times[0, T])\right. \\
u(x, 0)=\alpha_{\varepsilon}, u(x, T)=\beta_{\varepsilon} \\
\left.u_{x}(0, t)=u_{x}(L, t)=0\right\} .
\end{gathered}
$$

In this setting of the problem, we show the following limiting value of the minimum of the action functional as $\varepsilon$ tends to 0 .

Theorem 3.1. For the minimization problem (3.4), we have

$$
\lim _{\varepsilon \rightarrow 0} \delta S_{\varepsilon}^{\theta}=\min _{N \in \mathbb{N}}\left(c_{0} N+\frac{c_{0} L^{2}}{4 N T}+\theta L+\frac{\theta^{2} N T}{c_{0}}\right) .
$$

Moreover, the minimal action path is a linear function with respect to $t$.

Remark 3.2. A description of the action minimum for an equal double-well potential is given in [9] and [17]. We notice that taking $\theta=0, R H S$ of (3.6) agrees with the value of the equal well case which they obtained. The change of the speed up the moving interfaces appears in the propagation cost due to the effect of the different depth, while the nucleation cost $c_{0} N$ doesn't change.

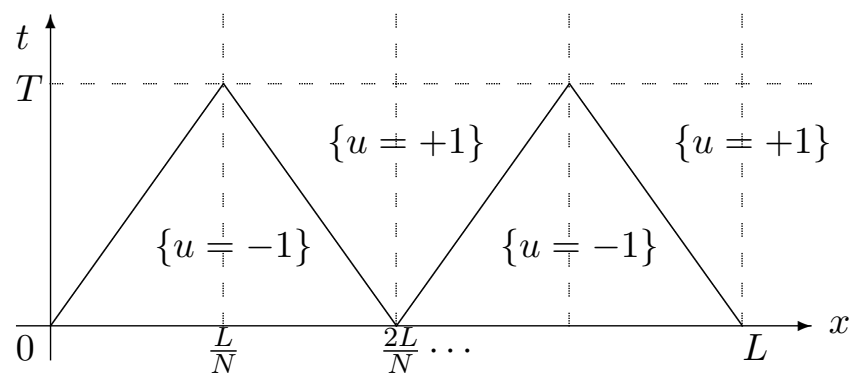

Remark 3.3. The number of interfaces $N$ does not depend on the sign of the constant $\theta$ since

$$
N \sim \sqrt{\frac{L^{2}}{4 T\left(1+\frac{\theta^{2}}{c_{0}^{2}} T\right)} .}
$$

We can also show the minimum for the opposite switching from the deeper stable state to the other,

$$
\lim _{\varepsilon \rightarrow 0} \delta S_{\varepsilon}^{\theta}=\min _{N \in \mathbb{N}}\left(c_{0} N+\frac{c_{0} L^{2}}{4 N T}-\theta L+\frac{\theta^{2} N T}{c_{0}}\right)
$$

by the slight change in our proof. Thus the cost is different but not the configuration for going up or down between the energy wells. 


\section{Upper Bounds}

In order to prove Theorem 3.1 we estimate the limsup and liminf of the minimal action functionals. In this section we consider the limsup by constructing the upper bound. We obtain the following.

Theorem 4.1. For the minimization problem of (3.4), we have

$$
\limsup _{\varepsilon \rightarrow 0} \delta S_{\varepsilon}^{\theta} \leq \min _{N \in \mathbb{N}}\left(c_{0} N+\frac{c_{0} L^{2}}{4 N T}+\theta L+\frac{\theta^{2} N T}{c_{0}}\right) .
$$

In order to prove it, we explictly construct a function $U$ which converges to the RHS of (4.1) as $\varepsilon$ goes to 0 . We show the following claim.

\section{Claim}

For arbitrary $\delta>0$, there exists a $\varepsilon_{0}$ such that if $\varepsilon<\varepsilon_{0}$, then

$$
\inf _{u \in \mathcal{A}_{\varepsilon}} S_{\varepsilon}^{\theta}(u) \leq \min _{N \in \mathbb{N}}\left(c_{0} N+\frac{c_{0} L^{2}}{4 N T}+\theta L+\frac{\theta^{2} N T}{c_{0}}\right)+\delta .
$$

While the outline of the construction is along the lines of that in [8], we have to use a different function as a transition profile. The effect of the difference of the well-depths will appear in the propagation cost which is connected in the speed of the propagating interfaces.

\section{Transition profile}

We use the solution $\phi_{\varepsilon}$ of the following ODE,

$$
\left\{\begin{array}{lr}
\lambda_{\varepsilon} \phi_{\varepsilon}^{\prime}=-\phi_{\varepsilon}^{\prime \prime}+W_{\varepsilon}^{\prime}\left(\phi_{\varepsilon}\right) & \text { in } \mathbb{R} \\
\lim _{x \rightarrow-\infty} \phi_{\varepsilon}(x)=\alpha_{\varepsilon}, & \lim _{x \rightarrow+\infty} \phi_{\varepsilon}(x)=\beta_{\varepsilon}
\end{array}\right.
$$

for the transition profile. We pick up the important facts which we need here. The existence of a unique pair of $\left(\phi_{\varepsilon}, \lambda_{\varepsilon}\right)$ is proved in [1]. According to [2], the explicit expression of the solution is given by

$$
\phi_{\varepsilon}(x)=\alpha_{\varepsilon}+\frac{\beta_{\varepsilon}-\alpha_{\varepsilon}}{1+\exp \left\{-\left(\beta_{\varepsilon}-\alpha_{\varepsilon}\right)\left(\frac{x}{\sqrt{2}}+\iota_{\varepsilon}\right)\right\}},
$$

where $\iota_{\varepsilon}$ is chosen as $\phi_{\varepsilon}(0)=\kappa_{\varepsilon}$, and

$$
\lambda_{\varepsilon}=\frac{1}{\sqrt{2}}\left(2 \kappa_{\varepsilon}-\alpha_{\varepsilon}-\beta_{\varepsilon}\right) .
$$

By associating

$$
\alpha_{\varepsilon} \approx-1-\frac{\theta \varepsilon}{2}, \quad \beta_{\varepsilon} \approx 1-\frac{\theta \varepsilon}{2} \quad \text { and } \quad \kappa_{\varepsilon} \approx \theta \varepsilon
$$


with (4.5), we notice the relation

$$
\lambda_{0}=\frac{2 \theta}{c_{0}} \quad \text { with } \quad \lambda_{0}:=\lim _{\varepsilon \rightarrow 0} \varepsilon^{-1} \lambda_{\varepsilon}
$$

between $\lambda_{\varepsilon}$ and $\theta$ holds in the limit as $\varepsilon$ goes to 0 . For the derivative of $\phi_{\varepsilon}$ it holds that

$$
\lim _{\varepsilon \rightarrow 0} \int_{-\infty}^{\infty}\left(\phi_{\varepsilon}^{\prime}(x)\right)^{2} d x=c_{0}
$$

Suppose that $N$ interfaces are created. We observe one nucleation point. Let $x_{0}$ be a nucleation point. Without loss of generality, we can assume $x_{0}=0$. We consider the action on $\left[-\frac{L}{N}, 0\right]$ and $\left[0, \frac{L}{N}\right]$, the neighborhood of the nucleation point. We set

$$
\Phi(x)= \begin{cases}\phi_{\varepsilon}\left(\frac{1}{\varepsilon}\left(L_{1}-x\right)\right) & \text { if } x \geq 0, \\ \phi_{\varepsilon}\left(\frac{1}{\varepsilon}\left(L_{1}+x\right)\right) & \text { if } x<0 .\end{cases}
$$

Here $L_{1}>0$ is a sufficiently small constant. For simplicity, we set $L_{1}=\sqrt{\varepsilon}$. Note that we need to regularize $\Phi$ at $x=0$ since the derivative of $\Phi$ is discontinuous.

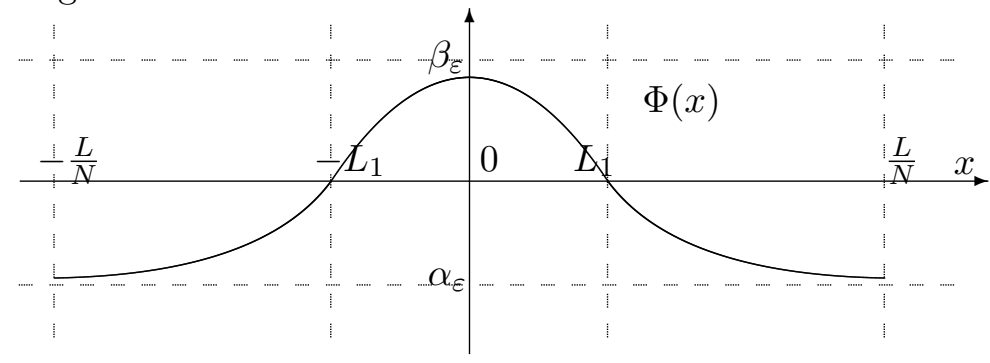

We briefly state recall the outline of the construction. We divide the interval $[0, T]$ into 5 parts called stages.

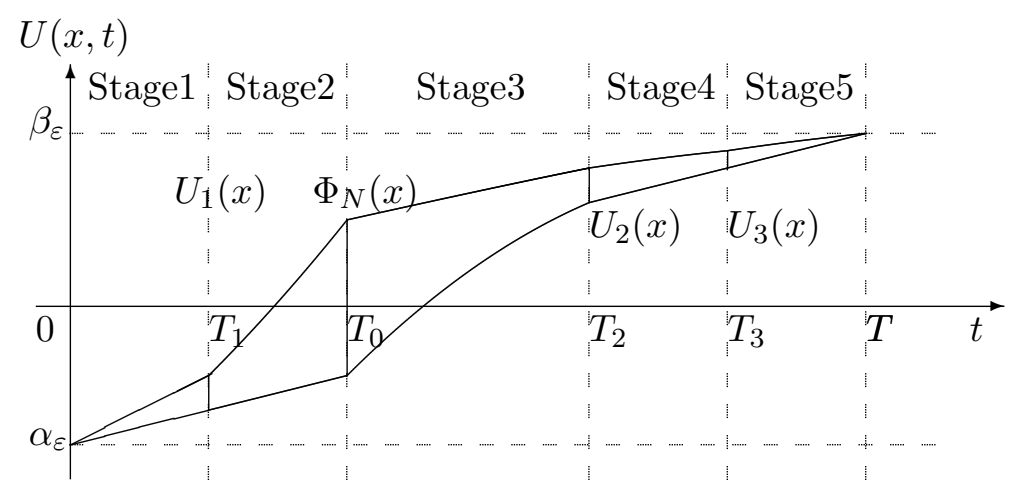

First, at some time $T_{0}$, we connect $\Phi(x)$ to the neighborhood of $\alpha_{\varepsilon}$ by using the solution of the time inverse PDE in Stage 2. We expect to obtain the nucleation cost of interfaces 
in this stage. Then we linearly interpolate to $\alpha_{\varepsilon}$ at the initial time in Stage 1. In Stage 3 , we propagate the interfaces by using a suitable linear function until it is close to the $\beta_{\varepsilon}$. The main idea is in this Stage 3. We have to pay attention to obtain the additional propagation cost from the effect of the unequal-wells. Then we connect the neighborhood of $\beta_{\varepsilon}$ by using a solution of PDE similarly to Stage 4 and again interpolate linearly in Stage 5 similarly to Stage 1 .

\section{Stage 2}

First we observe only one interface on $\left[0, \frac{L}{N}\right]$. We solve the equation $\sqrt{\varepsilon} u_{t}-\frac{1}{\sqrt{\varepsilon}} F_{\varepsilon}^{\theta}=0$ in reverse for time with the initial value $\Phi$ from some time $t=T_{0}$. We set $T_{0}=0$ for a while. Let $\widetilde{u}(x, t)=u(x,-t)$, we obtain

$$
\begin{cases}\widetilde{u}_{t}-\widetilde{u}_{x x}+\frac{W_{\varepsilon}^{\prime}(\widetilde{u})}{\varepsilon^{2}}=0 & \text { in }\left[0, \frac{L}{N}\right] \times \mathbb{R}^{+}, \\ \widetilde{u}(x, 0)=\Phi(x) & \text { in }\left[0, \frac{L}{N}\right] .\end{cases}
$$

According to Theorem 1 in [3], for sufficiently small $\varepsilon$ within time $t \approx \varepsilon^{2}|\log \varepsilon|$ we have

$$
\left\|\widetilde{u}-\alpha_{\varepsilon}\right\|_{L^{2}(0, L)}=o(\varepsilon) .
$$

Indeed, applying Theorem 1 in [3] to $\widetilde{u}$, there exist positive constants $\varepsilon_{1}>0, \tau_{0}>0$ and $M_{0}>0$ such that for any $\varepsilon \in\left(0, \varepsilon_{1}\right)$,

$$
\begin{gathered}
\alpha_{\varepsilon}-\varepsilon \leq \widetilde{u}(x, t) \leq \beta_{\varepsilon}+\varepsilon \quad \text { for } x \in\left[0, \frac{L}{N}\right], \quad t \geq \tau_{0} \varepsilon^{2}|\log \varepsilon|, \\
\widetilde{u}\left(x, \tau_{0} \varepsilon^{2}|\log \varepsilon|\right) \geq \beta_{\varepsilon}-\varepsilon \quad \text { for } x \in \Omega^{+} \\
\widetilde{u}\left(x, \tau_{0} \varepsilon^{2}|\log \varepsilon|\right) \leq \alpha_{\varepsilon}+\varepsilon \quad \text { for } x \in \Omega^{-}
\end{gathered}
$$

where

$$
\begin{aligned}
& \Omega^{+}=\left\{x \in\left[0, \frac{L}{N}\right]\left|\Phi(x)>\kappa_{\varepsilon}+M_{0} \varepsilon\right| \log \varepsilon \mid\right\}, \\
& \Omega^{-}=\left\{x \in\left[0, \frac{L}{N}\right]\left|\Phi(x)<\kappa_{\varepsilon}-M_{0} \varepsilon\right| \log \varepsilon \mid\right\} .
\end{aligned}
$$

By (4.4) we have $\Omega^{+}=\left[0, A_{\varepsilon}\right]$ and $\Omega^{-}=\left[B_{\varepsilon}, \frac{L}{N}\right]$ where $A_{\varepsilon}=o(\varepsilon)$ and $B_{\varepsilon}=o(\varepsilon)$. Thus at $t=\tau_{0} \varepsilon^{2}|\log \varepsilon|$ we obtain

$$
\begin{aligned}
\int_{0}^{\frac{L}{N}}\left|\widetilde{u}-\alpha_{\varepsilon}\right|^{2} d x & \leq \int_{0}^{A_{\varepsilon}}+\int_{A_{\varepsilon}}^{B_{\varepsilon}}+\int_{B_{\varepsilon}}^{\frac{L}{N}}\left|\widetilde{u}-\alpha_{\varepsilon}\right|^{2} d x \\
& \leq c A_{\varepsilon}+c B_{\varepsilon}+\varepsilon^{2} \frac{L}{N}=o(\varepsilon) .
\end{aligned}
$$

Thus (4.11) holds. The proof on $\left[-\frac{L}{N}, 0\right]$ is similar. 
Let $T_{1}=T_{0}-\tau_{0} \varepsilon^{2}|\log \varepsilon|$ and $u_{1}(x)=\widetilde{u}_{1}\left(x, T_{1}\right)$. We consider the action cost on this stage. Since there are $N$ interfaces, let $U_{1}$ and $\Phi_{N}$ be functions repeating the above construction connecting $u_{1}$ and $\Phi N$ times between $[0, L]$ and let $U$ be a function connecting $U_{1}$ and $\Phi_{N}$ using the solution of (4.10) in $\left[T_{1}, T_{0}\right]$. Since $U$ is a solution of $\sqrt{\varepsilon} U_{t}-\frac{1}{\sqrt{\varepsilon}} F_{\varepsilon}^{\theta}=0$ in $\left(0, \frac{L}{N}\right] \times\left[T_{1}, T_{0}\right)$ we have

$$
\begin{aligned}
\left.S_{\varepsilon}^{\theta}(U)\right|_{t=T_{1}} ^{T_{0}} & =\frac{1}{4} \int_{T_{1}}^{T_{0}} \int_{0}^{L}\left(\sqrt{\varepsilon} U_{t}-\frac{1}{\sqrt{\varepsilon}} F_{\varepsilon}^{\theta}\right)^{2}+4 U_{t} F_{\varepsilon}^{\theta} d x d t \\
& =\int_{T_{1}}^{T_{0}} \int_{0}^{L} U_{t} F_{\varepsilon}^{\theta} d x d t \\
& =\left.E_{\varepsilon}^{\theta}(U)\right|_{s=T_{0}}-\left.E_{\varepsilon}^{\theta}(U)\right|_{s=T_{1}} \\
& =E_{\varepsilon}^{\theta}\left(\Phi_{N}\right)-E_{\varepsilon}^{\theta}\left(U_{1}\right) .
\end{aligned}
$$

By (4.11), for sufficiently small $\varepsilon>0$, we have

$$
E_{\varepsilon}^{\theta}\left(U_{1}\right) \geq E_{\varepsilon}\left(U_{1}\right)+\int_{0}^{L} \theta U_{1} d x \geq-\theta L+o(\varepsilon) .
$$

For one interface, by scaling back $y=\frac{L_{1}-x}{\varepsilon}$ and since $W_{\varepsilon}(\Phi(y))=\frac{\left(\Phi^{\prime}(y)\right)^{2}}{2}+W_{\varepsilon}\left(\alpha_{\varepsilon}\right)+$ $\lambda_{\varepsilon} \int_{-\infty}^{y}\left(\Phi^{\prime}(s)\right) d s$ by (4.3), we have

$$
\begin{aligned}
\int_{0}^{\frac{L}{N}} \frac{\varepsilon\left(\Phi_{x}\right)^{2}}{2}+\frac{W_{\varepsilon}(\Phi)}{\varepsilon} d x & =\int_{\frac{L_{1}-\frac{L}{N}}{\varepsilon}}^{\frac{L_{1}}{\varepsilon}} \frac{\left(\Phi^{\prime}(y)\right)^{2}}{2}+W_{\varepsilon}(\Phi(y)) d y \\
& =\int_{\frac{L_{1}-\frac{L}{N}}{\varepsilon}}^{\frac{L_{1}}{\varepsilon}}\left(\Phi^{\prime}\right)^{2}+W_{\varepsilon}\left(\alpha_{\varepsilon}\right)+\lambda_{\varepsilon} \int_{-\infty}^{y}\left(\Phi^{\prime}(s)\right) d s d y .
\end{aligned}
$$

Since the interface moves by the speed $L_{1}=\sqrt{\varepsilon}$, the third term of (4.20) vanishes as $\varepsilon$ tends to 0 . Indeed, for an arbitrarily small constant $\eta>0$,

$$
\begin{aligned}
& \int_{\frac{L_{1}-\frac{L}{N}}{\varepsilon}}^{\frac{L_{1}}{\varepsilon}} \lambda_{\varepsilon} \int_{-\infty}^{y}\left(\Phi^{\prime}(s)\right) d s d y=\int_{\eta}^{L_{1}}+\int_{-\eta}^{\eta}+\int_{L_{1}-\frac{L}{N}}^{-\eta} \frac{\lambda_{\varepsilon}}{\varepsilon} \int_{-\infty}^{\varepsilon y}\left(\Phi^{\prime}(s)\right) d s d y \\
& \leq \frac{\lambda_{\varepsilon}}{\varepsilon}\left\{\left(L_{1}+2 \eta\right) \int_{-\infty}^{+\infty}\left(\Phi^{\prime}(s)\right) d s+\int_{-\infty}^{-\eta} \int_{-\infty}^{\varepsilon y}\left(\Phi^{\prime}(s)\right) d s d y\right\} .
\end{aligned}
$$

Let $\delta>0$ be an arbitrary constant and fixed. For sufficiently small $\varepsilon>0$, by (4.7) and (4.8), we have

$$
\int_{0}^{\frac{L}{N}} \frac{\varepsilon\left(\Phi_{x}\right)^{2}}{2}+\frac{W_{\varepsilon}(\Phi)}{\varepsilon} d x \leq c_{0}-\frac{\theta L}{N}+\delta
$$

Thus we obtain

$$
\left.E_{\varepsilon}^{\theta}\left(\Phi_{N}\right)\right|_{t=T_{1}} ^{T_{0}} \leq N c_{0}-\theta L+\delta .
$$


By (4.19) and (4.23), we obtain the action cost

$$
\left.S_{\varepsilon}^{\theta}(U)\right|_{t=T_{1}} ^{T_{0}} \leq \theta L+N c_{0}-\theta L+\delta=N c_{0}+\delta .
$$

\section{Stage 1}

We linearly interpolate from $U_{1}$ to $\alpha_{\varepsilon}$. We extend the lemma of Faris and Jona-Lasinio [6] to the unequal-well case in our scaling.

Lemma 4.2. For bounded functions $v_{1}(x)$ and $v_{2}(x)$ such that $\left\|\varepsilon^{-\frac{1}{2}} F_{\varepsilon}^{\theta}\left(v_{1}\right)\right\|_{L^{2}(0, L)}$ and $\left\|\varepsilon^{-\frac{1}{2}} F_{\varepsilon}^{\theta}\left(v_{2}\right)\right\|_{L^{2}(0, L)}$ are uniformly bounded, we define the linear interpolation $v(x, t)=$ $v_{1}(x)\left(1-\frac{t}{\tau}\right)+v_{2}(x) \frac{t}{\tau}$ for $0<t<\tau$. Then there exists a constant $c_{F}>0$ and a time $0<t_{F}<T$ such that the corresponding $v$ satisfies

$$
\left.S_{\varepsilon}^{\theta}(v)\right|_{t=0} ^{t_{F}} \leq c_{F} \varepsilon^{-\frac{1}{2}}\left\|v_{2}-v_{1}\right\|_{L^{2}(0, L)} .
$$

Proof. Let $t_{F}$ be fixed (we choose it later).

$$
\begin{aligned}
\left.S_{\varepsilon}^{\theta}(v)\right|_{t=0} ^{t_{F}} & =\frac{1}{4} \int_{0}^{t_{F}} \int_{0}^{L}\left(\sqrt{\varepsilon} v_{t}+\frac{1}{\sqrt{\varepsilon}} F_{\varepsilon}^{\theta}(v)\right)^{2} d x d t \\
& \leq \frac{1}{2} \int_{0}^{t_{F}} \int_{0}^{L} \varepsilon v_{t}^{2} d x d t+\frac{1}{2} \int_{0}^{t_{F}} \int_{0}^{L} \frac{1}{\varepsilon}\left\{F_{\varepsilon}^{\theta}(v)\right\}^{2} d x d t
\end{aligned}
$$

For the first term of (4.26), we have

$$
\begin{aligned}
\frac{1}{2} \int_{0}^{t_{F}} \int_{0}^{L} \varepsilon v_{t}^{2} d x d t & \leq \frac{\varepsilon}{2} \int_{0}^{t_{F}} \int_{0}^{L} \frac{1}{t_{F}^{2}}\left(v_{2}-v_{1}\right)^{2} d x d t \\
& \leq \frac{\varepsilon}{2 t_{F}}\left\|v_{2}-v_{1}\right\|_{L^{2}(0, L)}^{2} .
\end{aligned}
$$

Next we consider the second term of (4.26). We set $\widetilde{t_{F}}=\frac{t_{F}}{\varepsilon^{2}}$ and $\widetilde{L}=\frac{L}{\varepsilon}$. By scaling $\widetilde{v}(y, s)=\left(1-\frac{s}{t_{F}}\right) \widetilde{v_{1}}(y)+\frac{s}{t_{F}} \widetilde{v_{2}}(y)$ with $y=\frac{x}{\varepsilon}$ and $s=\frac{t}{\varepsilon^{2}}$, we have

$$
\frac{1}{2} \int_{0}^{t_{F}} \int_{0}^{L} \frac{1}{\varepsilon}\left\{F_{\varepsilon}^{\theta}(v)\right\}^{2} d x d t=\frac{1}{2} \int_{0}^{\widetilde{t_{f}}} \int_{0}^{\widetilde{L}}\left\{F^{\theta}\{\widetilde{v})\right\}^{2} d y d s
$$

where $F^{\theta}(\widetilde{v})=-\widetilde{v}_{y y}+W_{\varepsilon}^{\prime}(\widetilde{v})$. Hence there exists a constant $c_{F}>0$ and a bounded function $\rho(s)$ satisfying

$$
\int_{0}^{\widetilde{L}}\left\{F^{\theta}\{\widetilde{v})\right\}^{2} d y \leq\left\|F^{\theta}\left(\widetilde{v}_{1}\right)\right\|_{L^{2}(0, \widetilde{L})}^{2}+\left\|F^{\theta}\left(\widetilde{v}_{2}\right)\right\|_{L^{2}(0, \widetilde{L})}^{2}+\rho(s) \leq c_{F}^{2} .
$$

Thus we obtain

$$
\frac{1}{2} \int_{0}^{\widetilde{t}_{F}} \int_{0}^{\widetilde{L}}\left\{F^{\theta}(\widetilde{v})\right\}^{2} d y d s \leq \frac{c_{F}^{2} \widetilde{t_{F}}}{2}=\frac{c_{F}^{2} t_{F}}{2 \varepsilon^{2}} .
$$


Consequently by (4.27) and (4.30) we obtain

$$
\left.S_{\varepsilon}^{\theta}(v)\right|_{t=0} ^{t_{F}} \leq \frac{\varepsilon}{2 t_{F}}\left\|v_{2}-v_{1}\right\|_{L^{2}(0, L)}^{2}+\frac{c_{F}^{2} t_{F}}{2 \varepsilon^{2}}
$$

Here we choose $t_{F}=\frac{\varepsilon^{\frac{3}{2}}}{c_{F}}\left\|v_{2}-v_{1}\right\|_{L^{2}(0, L)}$, and the claim follows.

Applying Lemma 4.2 to $v_{1}=\alpha_{\varepsilon}$ and $v_{2}=U_{1}$, there exist a constant $c_{F}>0$ and a time $t_{F}>0$ such that

$$
\left.S_{\varepsilon}^{\theta}\left(U_{1}\right)\right|_{t=0} ^{t_{F}} \leq c_{F} \varepsilon^{-\frac{1}{2}}\left\|\alpha_{\varepsilon}-U_{1}\right\|_{L^{2}(0, L)} .
$$

By (4.11), for $\delta>0$ fixed before, we can choose $\varepsilon$ small enough to satisfy

$$
\varepsilon^{-\frac{1}{2}}\left\|\alpha_{\varepsilon}-U_{1}\right\|_{L^{2}(0, L)} \leq \frac{\delta}{c_{F}}
$$

Set $T_{0}=t_{F}+\tau_{0} \varepsilon^{2}|\log \varepsilon|$. Consequently, we obtain the action cost in Stage1,

$$
\left.S_{\varepsilon}^{\theta}(U)\right|_{t=0} ^{T_{1}} \leq \delta
$$

\section{Stage 3}

We denote the location of interfaces by $x= \pm\left(L_{1}-\varepsilon^{-1} \lambda_{\varepsilon} t+H(t)\right)$. We solve the variational problem what $H(s)$ minimizes the action functional later. Let

$$
v(x, t)= \begin{cases}\phi_{\varepsilon}\left(\frac{1}{\varepsilon}\left(L_{1}-\lambda_{\varepsilon} \varepsilon^{-1} t+H(t)-x\right)\right) & x \geq 0, \\ \phi_{\varepsilon}\left(\frac{1}{\varepsilon}\left(L_{1}-\lambda_{\varepsilon} \varepsilon^{-1} t+H(t)+x\right)\right) & x<0 .\end{cases}
$$

by using the solution $\phi_{\varepsilon}$ of ODE (4.3) and set

$$
u(x, t)=v\left(x, t-T_{0}\right) .
$$

Since

$$
u_{t}=v_{t}=\varepsilon^{-2}\left(-\lambda_{\varepsilon}+\varepsilon H^{\prime}(t)\right) \phi_{\varepsilon}^{\prime} \quad \text { and } \quad u_{x x}=v_{x x}=\varepsilon^{-2} \phi_{\varepsilon}^{\prime \prime}
$$

and $\phi_{\varepsilon}$ is a solution of ODE (4.3), we obtain

$$
\begin{aligned}
\left(\sqrt{\varepsilon} u_{t}+\frac{1}{\sqrt{\varepsilon}} F_{\varepsilon}^{\theta}\right)^{2} & =\varepsilon^{-3}\left\{\left(-\lambda_{\varepsilon}+\varepsilon H^{\prime}(t)\right) \phi_{\varepsilon}^{\prime}-\phi_{\varepsilon}^{\prime \prime}+W_{\varepsilon}^{\prime}\left(\phi_{\varepsilon}\right)\right\}^{2} \\
& =\varepsilon^{-1}\left(H^{\prime}(t) \phi_{\varepsilon}^{\prime}\right)^{2} .
\end{aligned}
$$

Let $t_{p}>0$ be fixed and we choose it later.

$$
\begin{aligned}
& \frac{1}{4} \int_{0}^{t_{p}} \int_{-\eta}^{\eta}\left(\sqrt{\varepsilon} u_{t}+\frac{1}{\sqrt{\varepsilon}} F_{\varepsilon}^{\theta}\right)^{2} d x d t \\
& \quad=\frac{1}{4 \varepsilon} \int_{0}^{t_{p}} \int_{-\eta}^{\eta}\left(H^{\prime}(t)\right)^{2}\left\{\phi_{\varepsilon}^{\prime}\left(\frac{1}{\varepsilon}\left(L_{1}-\lambda_{\varepsilon} \varepsilon^{-1} t+H(t)-x\right)\right)\right\}^{2} d x d t \\
& \quad \leq \frac{1}{4} \int_{0}^{t_{p}}\left(H^{\prime}(t)\right)^{2} d t \int_{-\infty}^{\infty}\left(\phi_{\varepsilon}^{\prime}(y)\right)^{2} d y
\end{aligned}
$$


Here we consider the variational problem of $\int_{0}^{t_{p}}\left(H^{\prime}(t)\right)^{2} d t$. For arbitrary $g \in C_{0}^{\infty}\left(0, t_{p}\right)$ and $l>0$ we can calculate the first variation

$$
\begin{aligned}
0 & =\left.\frac{\partial}{\partial l} \int_{0}^{t_{p}}\left(H^{\prime}(t)+l g^{\prime}(t)\right)^{2} d t\right|_{l=0} \\
& =2 \int_{0}^{t_{p}} H^{\prime}(t) g^{\prime}(t) d t \\
& =-2 \int_{0}^{t_{p}} H^{\prime \prime}(t) g(t) d t .
\end{aligned}
$$

By the fundamental lemma of calculus of variations, $H^{\prime \prime}(t)=0$. Thus, $H(t)$ is a linear function with respect to $t$. Let $t_{p}=T-2 T_{0}$. Since $N$ interfaces propagate over a length $L$ in time $t_{p}$, the velocity of interfaces is

$$
\pm \frac{L}{N t_{p}}
$$

On the other hand, since the location of interface is $x= \pm\left(L_{1}-\lambda_{\varepsilon} \varepsilon^{-1} t+H(t)\right)$, therefore

$$
x_{t}= \pm\left(-\lambda_{\varepsilon} \varepsilon^{-1}+H^{\prime}(t)\right) .
$$

By (4.41) and (4.42), we obtain

$$
H^{\prime}(t)=\lambda_{\varepsilon} \varepsilon^{-1}+\frac{L}{N t_{p}}
$$

By (4.39) and (4.43), for one interface we obtain

$$
\begin{aligned}
& \frac{1}{4} \int_{0}^{t_{p}} \int_{-\eta}^{\eta}\left(\sqrt{\varepsilon} u_{t}+\frac{1}{\sqrt{\varepsilon}} F_{\varepsilon}^{\theta}\right)^{2} d x d t \\
& \leq \frac{1}{4} \int_{0}^{t_{p}}\left(\lambda_{\varepsilon} \varepsilon^{-1}+\frac{L}{N t_{p}}\right)^{2} d t \int_{-\infty}^{\infty}\left(\phi_{\varepsilon}^{\prime}(y)\right)^{2} d y \\
& =\frac{t_{p}}{4}\left(\lambda_{\varepsilon} \varepsilon^{-1}+\frac{L}{N t_{p}}\right)^{2} \int_{-\infty}^{\infty}\left(\phi_{\varepsilon}^{\prime}(y)\right)^{2} d y .
\end{aligned}
$$

Since $t_{p}=T-2 T_{0}$ and (4.8), for $\delta>0$ fixed before, we have

$$
\frac{1}{4} \int_{0}^{t_{p}} \int_{-\eta}^{\eta}\left(\sqrt{\varepsilon} u_{t}+\frac{1}{\sqrt{\varepsilon}} F_{\varepsilon}^{\theta}\right)^{2} d x d t \leq \frac{T}{4}\left(\lambda_{0}+\frac{L}{N T}\right)^{2} c_{0}+\delta
$$

for sufficiently small $\varepsilon>0$. Let $T_{2}=T_{0}+t_{p}$. Since the number of interfaces is $N$, by (4.7) we obtain the action cost

$$
\left.S_{\varepsilon}^{\theta}(U)\right|_{s=T_{0}} ^{T_{2}} \leq \frac{N T}{4}\left(\frac{2 \theta}{c_{0}}+\frac{L}{N T}\right)^{2} c_{0}+\delta .
$$




\section{Stage 4}

We set $U_{2}(x)$ as the function $U$ of Stage 3 at time $T_{2}$. In a way similar to Stage 2, we use the solution of $\sqrt{\varepsilon} u_{t}+\frac{1}{\sqrt{\varepsilon}} F_{\varepsilon}^{\theta}=0$ with initial value $U_{2}$. By applying Theorem 1 in [3] again, there exists a $\tau_{0}^{\prime}<\tau_{0}$ satisfying $\varepsilon^{-\frac{1}{2}}\left\|U_{2}-\beta_{\varepsilon}\right\|_{L^{2}(0, L)} \leq \delta$ within time $t=\tau_{0}^{\prime} \varepsilon^{2}|\log \varepsilon|$. Moreover, the action cost is 0 in this stage since $u$ is the solution of $\sqrt{\varepsilon} u_{t}+\frac{1}{\sqrt{\varepsilon}} F_{\varepsilon}^{\theta}=0$.

\section{Stage 5}

We set $U_{3}(x)$ as the function of Stage 4 at time $T_{3}=T_{2}+\tau_{0}^{\prime} \varepsilon^{2}|\log \varepsilon|$. Let $U$ be a linearly interpolation from $U_{3}$ to $\beta_{\varepsilon}$. We obtain the action cost in Stage 5

$$
\left.S_{\varepsilon}^{\theta}(U)\right|_{t=T_{3}} ^{T} \leq \delta
$$

as in Stage 1.

Consequently the upper bound follows from Stage 1-5.

\section{Lower Bounds}

Next we show the lower bound of the action functional agrees with the upper bound constructed in Section 4. It is sufficient to consider a sequence $\left\{u_{\varepsilon}\right\}$ with $u_{\varepsilon} \in \mathcal{A}_{\varepsilon}$ which has uniformly bounded action.

Theorem 5.1. Let $\left\{u_{\varepsilon}\right\}$ be a sequence with $u_{\varepsilon} \in \mathcal{A}_{\varepsilon}$ which has uniformly bounded action. Then

$$
\liminf _{\varepsilon \rightarrow 0} S_{\varepsilon}^{\theta}\left(u_{\varepsilon}\right) \geq \min _{N \in \mathbb{N}}\left(c_{0} N+\frac{c_{0} L^{2}}{4 N T}+\theta L+\frac{\theta^{2} N T}{c_{0}}\right) .
$$

\subsection{Boundedness of Energies}

The upper bound of the action functional easily leads to a bound on the energy $E_{\varepsilon}^{\theta}$ and $L^{2}$-norm of $\frac{F_{\varepsilon}^{\theta}}{\sqrt{\varepsilon}}$. The uniform bound of $\left\{E_{\varepsilon}\right\}$ and $\left\{u_{\varepsilon}\right\}$ also follows from it. Let $\left\{u_{\varepsilon}\right\}$ be a sequence with $u_{\varepsilon} \in \mathcal{A}_{\varepsilon}$ which has uniformly bounded action and let $A_{0}$ be the upper bound of the action functional, that is, for sufficiently small $\varepsilon>0$,

$$
S_{\varepsilon}^{\theta}\left(u_{\varepsilon}\right) \leq A_{0} .
$$

By (1.6), for arbitrary time $s \in[0, T]$, we have

$$
\left.E_{\varepsilon}^{\theta}\left(u_{\varepsilon}\right)\right|_{t=s}-\left.E_{\varepsilon}^{\theta}\left(u_{\varepsilon}\right)\right|_{t=0} \leq S_{\varepsilon}^{\theta}\left(u_{\varepsilon}\right) .
$$

As $\alpha_{\varepsilon} \approx-1-\frac{\varepsilon \theta}{2}$, the energy at $t=0$ is

$$
\left.E_{\varepsilon}^{\theta}\left(u_{\varepsilon}\right)\right|_{t=0}=E_{\varepsilon}^{\theta}\left(\alpha_{\varepsilon}\right)=\int_{0}^{L} \frac{W_{\varepsilon}\left(\alpha_{\varepsilon}\right)}{\varepsilon} d x=(-\theta+o(\varepsilon)) L .
$$


Thus we obtain the bound on the energy

$$
\left.E_{\varepsilon}^{\theta}\left(u_{\varepsilon}\right)\right|_{t=s} \leq A_{0}+(-\theta+o(\varepsilon)) L \leq A_{0}
$$

for sufficient small $\varepsilon>0$. Moreover, since

$$
A_{0} \geq S_{\varepsilon}^{\theta}\left(u_{\varepsilon}\right) \geq \frac{1}{4} \int_{0}^{T} \int_{0}^{L} \frac{\left(F_{\varepsilon}^{\theta}\right)^{2}}{\varepsilon} d x d t+\left.E_{\varepsilon}^{\theta}\left(u_{\varepsilon}\right)\right|_{t=T}-\left.E_{\varepsilon}^{\theta}\left(u_{\varepsilon}\right)\right|_{t=0}
$$

and

$$
\left.E_{\varepsilon}^{\theta}\left(u_{\varepsilon}\right)\right|_{t=T}=E_{\varepsilon}^{\theta}\left(\beta_{\varepsilon}\right) \geq 0,\left.\quad E_{\varepsilon}^{\theta}\left(u_{\varepsilon}\right)\right|_{t=0}<0
$$

we obtain

$$
A_{0} \geq \frac{1}{4} \int_{0}^{T} \int_{0}^{L} \frac{\left(F_{\varepsilon}^{\theta}\right)^{2}}{\varepsilon} d x d t
$$

By taking liminf with respect to $\varepsilon$ and using Fatou's lemma,

$$
A_{0} \geq \frac{1}{4} \int_{0}^{T} \liminf _{\varepsilon \rightarrow 0} \int_{0}^{L} \frac{\left(F_{\varepsilon}^{\theta}\right)^{2}}{\varepsilon} d x d t .
$$

Thus we can take a subsequence $\left\{\varepsilon_{i}\right\}$ for an arbitrary fixed time $s \in[0, T]$ satisfying

$$
\int_{0}^{L} \frac{\left(F_{\varepsilon_{i}}^{\theta}\right)^{2}}{\varepsilon_{i}} d x \leq A_{0}
$$

Next we show the uniform bound of the energy $\left\{E_{\varepsilon}\right\}$ and $\left\{u_{\varepsilon}\right\}$.

Lemma 5.2. Let $\left\{u_{\varepsilon}\right\}$ be a sequence with $u_{\varepsilon} \in \mathcal{A}_{\varepsilon}$ which has a uniformly bounded action. Then $\left\{u_{\varepsilon}\right\}$ and $\left\{E_{\varepsilon}\left(u_{\varepsilon}\right)\right\}$ are uniformly bounded for sufficiently small $\varepsilon>0$.

Proof. We restrict to $\left\{u_{\varepsilon} \geq 1\right\}$. Since there exists a constant $c>0$ such that $W\left(u_{\varepsilon}\right) \geq$ $c\left(u_{\varepsilon}-1\right)^{2}$,

$$
A_{0} \geq \int_{\left\{u_{\varepsilon} \geq 1\right\}} \frac{W\left(u_{\varepsilon}\right)}{\varepsilon}+\theta u_{\varepsilon} d x \geq \int_{\left\{u_{\varepsilon} \geq 1\right\}} \frac{c\left(u_{\varepsilon}-1\right)^{2}}{4 \varepsilon}-\theta\left|u_{\varepsilon}\right| d x .
$$

Since there exists a $\widetilde{c}=\widetilde{c}(\theta)>0$ such that

$$
\frac{c\left(u_{\varepsilon}-1\right)^{2}}{4 \varepsilon}-\theta\left|u_{\varepsilon}\right| \geq \frac{c\left(u_{\varepsilon}-1\right)^{2}}{4 \varepsilon}-\theta\left|u_{\varepsilon}\right|^{2} \geq \frac{\widetilde{c}}{8 \varepsilon} u_{\varepsilon}^{2}
$$

and (5.11), we obtain the bound on the $L^{2}$-norm of $u_{\varepsilon}$

$$
\int_{\left\{u_{\varepsilon} \geq 1\right\}} u_{\varepsilon}^{2} d x \leq \frac{8 A_{0}}{\tilde{c}} \varepsilon
$$


We can obtain the estimate of $u_{\varepsilon}$ by using a composite function. We set

$$
\Phi(s)=\int_{0}^{s} \sqrt{\frac{W(y)}{2}} d y .
$$

We use the estimate of BV-norm of $\Phi$.

$$
\begin{aligned}
\int\left(\Phi\left(u_{\varepsilon}\right)\right)_{x} d x & =\int \Phi^{\prime}\left(u_{\varepsilon}\right)\left|u_{\varepsilon x}\right| d x=\int \sqrt{\frac{W\left(u_{\varepsilon}\right)}{2}}\left|u_{\varepsilon x}\right| d x \\
& \leq \int \frac{\varepsilon\left(u_{\varepsilon x}\right)^{2}}{2}+\frac{W\left(u_{\varepsilon}\right)}{\varepsilon} d x \\
& \leq \int \frac{\varepsilon\left(u_{\varepsilon x}\right)^{2}}{2}+\frac{W_{\varepsilon}\left(u_{\varepsilon}\right)}{\varepsilon}+\theta L \int u_{\varepsilon}^{2} d x \\
& \leq A_{0}+\frac{8 \theta L A_{0}}{\tilde{c}} \varepsilon \leq 2 A_{0} .
\end{aligned}
$$

Thus the BV-norm of $\Phi$ is bounded. Since for arbitrary $x, y \in[0, L]$,

$$
\left|\Phi\left(u_{\varepsilon}(x)\right)-\Phi\left(u_{\varepsilon}(y)\right)\right| \leq \int_{x}^{y}\left(\Phi\left(u_{\varepsilon}\right)\right)_{x} d x \leq 2 A_{0},
$$

it is sufficient to find a point $x_{0} \in[0, L]$ where $\Phi\left(u_{\varepsilon}\left(x_{0}\right)\right)$ is bounded. Since

$$
\int_{\left\{u_{\varepsilon} \geq 1\right\}}\left|u_{\varepsilon}\right| d x \leq \int_{\left\{u_{\varepsilon} \geq 1\right\}}\left|u_{\varepsilon}\right|^{2} d x \leq \frac{8 A_{0} L^{2}}{\tilde{c}} \varepsilon
$$

by (5.13), there exists $A_{1}>0$ and $x_{0} \in[0, L]$ such that

$$
\left|u_{\varepsilon}\left(x_{0}\right)\right| \leq A_{1}
$$

By (5.16) and (5.18),

$$
\Phi\left(u_{\varepsilon}(x)\right) \leq \Phi\left(u_{\varepsilon}\left(x_{0}\right)\right)+\Phi\left(A_{1}\right) \leq 2 A_{0}+\Phi\left(A_{1}\right) .
$$

Since

$$
\int_{0}^{u_{\varepsilon}(x)} \sqrt{\frac{W(y)}{2}} d y \leq 2 A_{0}+\Phi\left(A_{1}\right)
$$

and $\sqrt{\frac{W(y)}{2}}(\geq 0)$ is a quadratic function, $u_{\varepsilon}(x)$ is bounded. Thus $E_{\varepsilon}\left(u_{\varepsilon}\right)$ is also bounded. 


\subsection{Definition of Measures and Lemmata}

Let $\left\{u_{\varepsilon}\right\}$ be a sequence with $u_{\varepsilon} \in \mathcal{A}_{\varepsilon}$ which has uniformly bounded action. We define the action measure by

$$
d \nu_{\varepsilon}:=\frac{1}{4}\left\{\varepsilon\left(u_{\varepsilon t}\right)^{2}+\frac{\left(F_{\varepsilon}^{\theta}\right)^{2}}{\varepsilon}\right\} d x d t .
$$

We define the space-time energy measure by

$$
d \widetilde{\mu}_{\varepsilon}:=d \mu_{\varepsilon}+d \eta_{\varepsilon}=\frac{\varepsilon}{2}\left(u_{\varepsilon x}\right)^{2}+\frac{W_{\varepsilon}\left(u_{\varepsilon}\right)}{\varepsilon} d x d t,
$$

where

$$
d \mu_{\varepsilon}:=\frac{\varepsilon}{2}\left(u_{\varepsilon x}\right)^{2}+\frac{W\left(u_{\varepsilon}\right)}{\varepsilon} d x d t \quad \text { and } \quad d \eta_{\varepsilon}:=\theta u_{\varepsilon} d x d t .
$$

We also define the energy measure at time $t$ by

$$
d \widetilde{\mu}_{\varepsilon}^{t}:=d \mu_{\varepsilon}^{t}+d \eta_{\varepsilon}^{t}=\frac{\varepsilon}{2}\left(u_{\varepsilon x}\right)^{2}+\frac{W_{\varepsilon}\left(u_{\varepsilon}\right)}{\varepsilon} d x
$$

where

$$
d \mu_{\varepsilon}^{t}:=\frac{\varepsilon}{2}\left(u_{\varepsilon x}\right)^{2}+\frac{W\left(u_{\varepsilon}\right)}{\varepsilon} d x \quad \text { and } \quad d \eta_{\varepsilon}^{t}:=\theta u_{\varepsilon} d x .
$$

We state important lemmata required to show the lower bound. We can prove them by the slight modification of [8] since the order of the additional term which comes from the difference of the well-depths is $\varepsilon$, which is sufficient to show the convergence of measures. The first lemma states the convergence of the sequence $\left\{u_{\varepsilon}\right\}$.

Lemma 5.3. Let $\left\{u_{\varepsilon}\right\}$ be a sequence with $u_{\varepsilon} \in \mathcal{A}_{\varepsilon}$ which has uniformly bounded action. Then there is a subsequence $\left\{u_{\varepsilon_{i}}\right\}$ which converges almost everywhere and in every $L^{p}$ to a limit $u_{0}$. Furthermore, $u_{0}= \pm 1$ almost everywhere in space for every time.

Next lemma implies that the limit energy of $\widetilde{\mu}_{\varepsilon}^{t}$ is obtained as the sum of the limit energy for the equal-well case and the term representing the effect of the different welldepths, that is, the difference of $\left|\left\{u_{0}=+1\right\}\right|$ and $\left|\left\{u_{0}=-1\right\}\right|$ except at the nucleation time.

Lemma 5.4. Let $\left\{u_{\varepsilon}\right\}$ be an arbitrary sequence with $u_{\varepsilon} \in \mathcal{A}_{\varepsilon}$ which has uniformly bounded action. By the weak star compactness of bounded measures, there exists a subsequence $\left\{\varepsilon_{i}\right\}$ satisfying $\widetilde{\mu}_{\varepsilon_{i}} \rightarrow \widetilde{\mu}$ and $\nu_{\varepsilon_{i}} \rightarrow \nu$ in the sense of Radon measure. For this subsequence, there exists a finite set of singular times $F_{\text {sing }}=\left\{T_{1}, T_{2}, \cdots, T_{M}\right\}$ such that for all $t \in$ $[0, T] \backslash F_{\text {sing }}$,

$$
\int \Psi d \widetilde{\mu}=\iint \Psi d \mu^{t}+\int \Psi d \eta^{t} d t \quad \text { for all } \Psi \in C([0, L] \times[0, T])
$$


and

$$
\eta_{\varepsilon_{i}}^{t} \rightarrow \eta^{t}=\theta u_{0} d x
$$

and

$$
\mu_{\varepsilon_{i}}^{t} \rightarrow \mu^{t}=\sum_{l=1}^{M} c_{0} m\left(x_{l}\right) \delta_{\left\{x_{l}\right\}} \text { with } m\left(x_{l}\right) \in \mathbb{N}
$$

as $\varepsilon_{i} \rightarrow 0$, where $\delta_{\left\{x_{l}\right\}}$ is the delta-measure at $x_{l}$. Furthermore $m\left(x_{l}\right)$ and $x_{l}$ are continuous in time.

Remark 5.5. We assume the "single" multiplicity of the limit interface measure $\mu^{t}$, namely $\left|m\left(x_{l}\right)\right|=1$ in Lemma 5.4. We need this assumption in (5.49) and (5.62).

Proof. Since the treatment of the part $\frac{\varepsilon_{i}}{2}\left(u_{\varepsilon_{i} x}\right)+\frac{W\left(u_{\varepsilon_{i}}\right)}{\varepsilon_{i}}$ of energy measure is essentially the same as the equal-well case, we only give a short sketch of the proof by following the discussion of Theorem 1.1 and 1.2 in [9]. Since $\left\{\widetilde{\mu}_{\varepsilon}\right\}$ is uniformly bounded, $\left\{\mu_{\varepsilon}\right\}$ and $\left\{\eta_{\varepsilon}\right\}$ are also bounded measures. We take subsequence $\widetilde{\mu}_{\varepsilon_{i}}, \mu_{\varepsilon_{i}}, \eta_{\varepsilon_{i}}$ and $\nu_{\varepsilon_{i}}$ which converge to measures $\widetilde{\mu}, \mu, \eta$ and $\nu$ in the sense of Radon measure respectively. Let $S:=\left\{\int_{0}^{L} d \nu\right.$ have a point mass $\} \subset[0, T]$. For a dense set $\left\{t_{j}\right\}_{j=1}^{\infty} \subset S^{c}$ by the diagonalization argument for $\mu_{\varepsilon_{i}}^{t_{j}}$ and $\eta_{\varepsilon_{i}}^{t_{j}}$, we can take a subsequence which converges to $\mu^{t_{j}}$ and $\eta^{t_{j}}$. Then we set $\mu^{t}:=\lim _{j \rightarrow \infty} \mu^{t_{j}}$ and $\eta^{t}:=\lim _{j \rightarrow \infty} \eta^{t_{j}}$, which are well-defined for all $t \in S^{c}$ and we set $\widetilde{\mu}^{t}:=\mu^{t}+\eta^{t}$. Indeed, for $\phi \in W^{1, \infty}([0, L])$ with $\|\phi\|_{W^{1, \infty}} \leq 1$ and for $t \in S^{c}$ by Lemma 5.3 , we have

$$
\int_{0}^{L} \phi d \eta_{\varepsilon_{i}}^{t}=\theta \int_{0}^{L} \phi u_{\varepsilon_{i}} d x \rightarrow \theta \int_{0}^{L} u_{0}(x, t) \phi d x \quad \text { as } i \rightarrow \infty .
$$

For $t, s \in S^{c}$ we have

$$
\left|\int_{0}^{L} \phi d \eta_{\varepsilon_{i}}^{t}-\int_{0}^{L} \phi d \eta_{\varepsilon_{i}}^{s}\right|=\theta\left|\int_{0}^{L}\left\{u_{\varepsilon_{i}}(x, t)-u_{\varepsilon_{i}}(x, s)\right\} \phi d x\right|
$$

and

$$
\begin{aligned}
& \int_{0}^{L} \phi d \mu_{\varepsilon_{i}}^{t}-\int_{0}^{L} \phi d \mu_{\varepsilon_{i}}^{s} \\
& \leq \int_{0}^{L} \phi d \widetilde{\mu}_{\varepsilon_{i}}^{t}-\int_{0}^{L} \phi d \widetilde{\mu}_{\varepsilon_{i}}^{s}-\left(\int_{0}^{L} \phi d \eta_{\varepsilon_{i}}^{t}-\int_{0}^{L} \phi d \eta_{\varepsilon_{i}}^{s}\right) \\
& \leq \frac{|s-t|}{2} \sup _{t \in[0, T]}\left[\int_{0}^{L} \varepsilon_{i}\left(u_{\varepsilon_{i} x}\right)^{2}\right]+\int_{s}^{t} \int_{0}^{L}\left\{\varepsilon_{i}\left(u_{\varepsilon_{i}}\right)^{2}+\frac{\left(F_{\varepsilon_{i}}^{\theta}\right)^{2}}{\varepsilon_{i}}\right\} d x d t \\
& \quad+\theta\left|\int_{0}^{L}\left\{u_{\varepsilon_{i}}(x, t)-u_{\varepsilon_{i}}(x, s)\right\} \phi d x\right| .
\end{aligned}
$$


By letting $i \rightarrow \infty$ and $j \rightarrow \infty$, the limit measure $\mu^{t}$ and $\eta^{t}$ are uniquely determined. Moreover from above calculation, $\mu^{t}:=\lim _{i \rightarrow \infty} \mu_{\varepsilon_{i}}^{t}, \eta^{t}:=\lim _{i \rightarrow \infty} \eta_{\varepsilon_{i}}^{t}$ are also well-defined for all $t \in S^{c}$. We set $\widetilde{\mu}^{t}:=\mu^{t}+\eta^{t}$. By Lebesgue's dominated convergence theorem, $\int \phi d \mu=\lim _{i \rightarrow \infty} \int \phi d \mu_{\varepsilon_{i}}+d \eta_{\varepsilon_{i}}=\lim _{i \rightarrow \infty} \iint \phi d \mu_{\varepsilon_{i}}^{t}+d \eta_{\varepsilon_{i}}^{t} d t=\iint \phi d \mu^{t}+d \eta^{t} d t$. Thus we obtain (5.26).

Since $W_{\varepsilon}^{\prime \prime}(s)=W^{\prime \prime}(s) \geq \kappa$ for $|s|>\frac{3}{4}$, by a slight modification of Theorem 2.1 in [9], we obtain a uniform bound on the discrepancy $\left|\frac{\varepsilon_{i}}{2}\left(u_{\varepsilon_{i} x}\right)^{2}-\frac{W\left(u_{\varepsilon_{i}}\right)}{\varepsilon_{i}}\right|$. Since $\alpha_{\varepsilon}=-1+o(\varepsilon)$ and $\beta_{\varepsilon}=1+o(\varepsilon)$, using an argument similar to Lemmas 2.1, 2.2 in [9], on the set $\left\{\left|u_{\varepsilon_{i}}\right|>1-s\right\}$ for a suitable $s$, which implies on the set $\left\{\left|u_{\varepsilon_{i}}\right|>1-s\right\}$ is far from transition layers, the energy $E\left(u_{\varepsilon_{i}}\right)$ is very small. Thus by separating the energy on the set from that on or near the transition layers and by the continuity in time, we obtain (5.28). The choice of a singular set from $S$ is similar to proof of Theorem 1.2 in [9].

We need the following lemma to estimate the propagation cost in the proof.

Lemma 5.6. Let $\left\{u_{\varepsilon_{i}}\right\}$ be an arbitrary sequence with $u_{\varepsilon_{i}} \in \mathcal{A}_{\varepsilon_{i}}$ which have uniformly bounded action. Then there exists a constant $c>0$ such that for any time $t, s$ for which $u_{\varepsilon_{i}}(\cdot, t) \rightarrow u_{0}(\cdot, t)$ and $u_{\varepsilon_{i}}(\cdot, s) \rightarrow u_{0}(\cdot, s)$ in $L^{3}$, we have

$$
|I(t ; r, x)-I(s ; r, x)| \leq c|t-s|^{\frac{1}{2}}
$$

for

$$
I(t ; r, x)=\int_{x-r}^{x+r}\left(u_{0}-\frac{u_{0}^{3}}{3}\right)(\cdot, t) d x .
$$

\subsection{Proof of Theorem 5.1}

In this section we give the lower bound of the action functional. Since we consider the nucleation cost and the propagation cost of interfaces separately, we divide $[0, T]$ into two parts, the neighborhood of nucleation points and the rest.

Proof. $S_{\varepsilon}^{\theta}\left(u_{\varepsilon}\right)$ converges to a constant with a limit defined to be $S_{0}^{\theta}$. By Lemma 5.4 and Lemma 5.3 , we choose a subsequence of $\left\{\varepsilon_{i}\right\}$ satisfying $\widetilde{\mu}_{\varepsilon_{i}} \rightarrow \widetilde{\mu}$ as Radon measure and $u_{\varepsilon_{i}} \rightarrow u_{0}$ a.e. and in $L^{3}[0, L]$ for all $t \in[0, T]$.

Let $F_{\text {sing }}=\left\{T_{1}, T_{2}, \cdots, T_{M}\right\}$ be a set of singular times in Lemma 5.4 with $T_{1}=0$ and $T_{M}=T$.

We consider the $\delta$-neighborhood of $F_{\text {sing }}$ and later let $\delta$ tend to 0 . We choose $\delta$ satisfying $\delta<\min _{k \neq j}\left|T_{j}-T_{k}\right|$ and

$$
A_{\delta}=\bigcup_{j=1}^{M}\left(\left[T_{j}-\delta, T_{j}+\delta\right] \cap[0, T]\right) .
$$


We set

$$
S_{\text {nuc. } \varepsilon_{i}}^{\theta, \delta}\left(u_{\varepsilon_{i}}\right)=\frac{1}{4} \int_{A_{\delta}} \int_{0}^{L}\left(\sqrt{\varepsilon_{i}}\left(u_{\varepsilon_{i}}\right)_{t}+\frac{F_{\varepsilon_{i}}^{\theta}}{\sqrt{\varepsilon_{i}}}\right)^{2} d x d t
$$

and

$$
S_{\text {prop. } \varepsilon_{i}}^{\theta, \delta}\left(u_{\varepsilon_{i}}\right)=\frac{1}{4} \int_{[0, T] \backslash A_{\delta}} \int_{0}^{L}\left(\sqrt{\varepsilon_{i}}\left(u_{\varepsilon_{i}}\right)_{t}+\frac{F_{\varepsilon_{i}}^{\theta}}{\sqrt{\varepsilon_{i}}}\right)^{2} d x d t .
$$

Formally the next calculation follows,

$$
\begin{aligned}
S_{0}^{\theta} & =\lim _{i \rightarrow \infty} S_{\varepsilon_{i}}^{\theta}\left(u_{\varepsilon_{i}}\right) \\
& =\lim _{i \rightarrow \infty}\left\{S_{\text {nuc. } \varepsilon_{i}}^{\theta, \delta}\left(u_{\varepsilon_{i}}\right)+S_{\text {prop. } \varepsilon_{i}}^{\theta, \delta}\left(u_{\varepsilon_{i}}\right)\right\} \\
& \geq \liminf _{i \rightarrow \infty} S_{\text {nuc. } \varepsilon_{i}}^{\theta, \delta}\left(u_{\varepsilon_{i}}\right)+\liminf _{i \rightarrow \infty} S_{\text {prop. } \varepsilon_{i}}^{\theta, \delta}\left(u_{\varepsilon_{i}}\right) \\
& =S_{\text {nuc. }}^{\theta, \delta}+S_{\text {prop. }}^{\theta, \delta} .
\end{aligned}
$$

Let $\delta$ tend to 0 ,

$$
S_{0}^{\theta} \geq \lim _{\delta \rightarrow 0}\left(S_{\text {nuc. }}^{\theta, \delta}+S_{\text {prop. }}^{\theta, \delta}\right)=S_{\text {nuc. }}^{\theta}+S_{\text {prop. }}^{\theta} .
$$

(Nucleation cost) First we consider the nucleation cost. On $\left[T_{j}-\delta, T_{j}+\delta\right]$ for $j \neq 1$ and $j \neq M$, by Lemma 5.4 we have

$$
\begin{aligned}
& \lim _{i \rightarrow \infty}\left\{\widetilde{\mu}_{\varepsilon_{i}}^{T_{j}+\delta}([0, L])-\widetilde{\mu}_{\varepsilon_{i}}^{T_{j}-\delta}([0, L])\right\} \\
& =\mu^{T_{j}+\delta}([0, L])-\mu^{T_{j}-\delta}([0, L])+\eta^{T_{j}+\delta}([0, L])-\eta^{T_{j}-\delta}([0, L]) .
\end{aligned}
$$

For $j=1$, (that is $t=0$ ) since

$$
\lim _{i \rightarrow \infty} \widetilde{\mu}_{\varepsilon_{i}}^{0}([0, L])=\lim _{i \rightarrow \infty} \int_{0}^{L} \frac{W_{\varepsilon_{i}}\left(\alpha_{\varepsilon_{i}}\right)}{\varepsilon_{i}} d x=-\theta L,
$$

we obtain

$$
\lim _{i \rightarrow \infty}\left\{\widetilde{\mu}_{\varepsilon_{i}}^{\delta}([0, L])-\widetilde{\mu}_{\varepsilon_{i}}^{0}([0, L])\right\}=\mu^{\delta}([0, L])+\eta^{\delta}([0, L])+\theta L .
$$

Let $\left(T_{J}, T_{J+1}\right)$ be an interval where $\mu^{t}([0, L])$ attains its maximum. Under the assumption of the single multiplicity of $\mu^{t}([0, L])$ in Remark 5.5 we can set

$$
\max _{t \in\left(T_{J}, T_{J+1}\right)} \mu^{t}([0, L])=\max _{t \in F_{\text {sing. }}^{c}} \mu^{t}([0, L]):=c_{0} N
$$

for some $N \in \mathbb{N}$. Since

$$
\bigcup_{j=1}^{J}\left(\left[T_{j}-\delta, T_{j}+\delta\right] \cap[0, T]\right) \subseteq A_{\delta}
$$


and the action functional is positive on an arbitrary interval of time, we obtain

$$
\begin{aligned}
S_{\text {nuc. }}^{\theta, \delta} & \geq \lim _{i \rightarrow \infty} \frac{1}{4} \int_{\bigcup_{j=1}^{J}\left[T_{j}-\delta, T_{j}+\delta\right] \cap[0, T]} \int_{0}^{L}\left(\sqrt{\varepsilon_{i}}\left(u_{\varepsilon_{i}}\right) t+\frac{F_{\varepsilon_{i}}^{\theta}}{\sqrt{\varepsilon_{i}}}\right)^{2} d x d t \\
& \geq \lim _{i \rightarrow \infty} \sum_{j=2}^{J}\left\{\widetilde{\mu}_{\varepsilon_{i}}^{T_{j}+\delta}([0, L])-\widetilde{\mu}_{\varepsilon_{i}}^{T_{j}-\delta}([0, L])\right\}+\lim _{i \rightarrow \infty}\left\{\widetilde{\mu}_{\varepsilon_{i}}^{\delta}([0, L])-\widetilde{\mu}_{\varepsilon_{i}}^{0}([0, L])\right\} .
\end{aligned}
$$

By (5.39) and (5.41), we obtain

$$
\begin{aligned}
S_{\text {nuc. }}^{\theta, \delta} \geq \sum_{j=2}^{J} & \left\{\mu^{T_{j}+\delta}([0, L])-\mu^{T_{j}-\delta}([0, L])+\eta^{T_{j}+\delta}([0, L])-\eta^{T_{j}-\delta}([0, L])\right\} \\
& +\mu^{\delta}([0, L])+\eta^{\delta}([0, L])+\theta L .
\end{aligned}
$$

Since $\mu^{t}([0, L])$ is a constant for $t \in\left(T_{j}, T_{j+1}\right)$ according to Lemma 5.4,

$$
\begin{aligned}
S_{\text {nuc. }}^{\theta, \delta} & \geq \mu^{T_{J}+\delta}([0, L])+\sum_{j=2}^{J}\left\{\eta^{T_{j}+\delta}([0, L])-\eta^{T_{j}-\delta}([0, L])\right\} \\
& +\mu^{\delta}([0, L])+\eta^{\delta}([0, L])+\theta L .
\end{aligned}
$$

Let $\delta$ tend to 0 in (5.46). Since

$$
\begin{gathered}
\sum_{j=2}^{J}\left\{\eta^{T_{j}+\delta}([0, L])-\eta^{T_{j}-\delta}([0, L])\right\} \rightarrow 0, \\
\mu^{\delta}([0, L]) \rightarrow 0 \quad \text { and } \quad \eta^{\delta}([0, L]) \rightarrow-\theta L
\end{gathered}
$$

as $\delta$ goes to 0 , by (5.42) we obtain the nucleation cost

$$
S_{n u c .}^{\theta} \geq c_{0} N
$$

(Propagation cost) Next we consider the propagation cost. We use the functional

$$
I_{[s, t]}(u):=\int_{s}^{t} \int_{0}^{L} u_{t}\left(1-u^{2}\right) d x d t .
$$

We use the fact that

$$
\lim _{i \rightarrow \infty} I_{\left[T_{j}+\delta, T_{j+1}-\delta\right]}\left(u_{\varepsilon_{i}}\right)=\left.\int_{0}^{L}\left\{u_{0}-\frac{\left(u_{0}\right)^{3}}{3}\right\} d x\right|_{t=T_{j}+\delta} ^{T_{j+1}-\delta}=\frac{2}{3} \Delta m_{j}^{\delta},
$$

where

$$
\Delta m_{j}^{\delta}=\theta^{-1}\left\{\eta^{T_{j+1}-\delta}([0, L])-\eta^{T_{j}+\delta}([0, L])\right\}
$$


We note that

$$
\lim _{\delta \rightarrow 0} \sum_{j=1}^{M-1} \Delta m_{j}^{\delta}=\sum_{j=1}^{M-1} \Delta m_{j}^{0}=2 L .
$$

By Hölder's inequality, we obtain

$$
\begin{aligned}
& I_{\left[T_{j}+\delta, T_{j+1}-\delta\right]}\left(u_{\varepsilon_{i}}\right) \\
& =\int_{T_{j}+\delta}^{T_{j+1}-\delta} \int_{0}^{L} u_{\varepsilon_{i} t}\left(1-u_{\varepsilon_{i}}^{2}\right) d x d t \\
& \leq 2\left\{\int_{T_{j}+\delta}^{T_{j+1}-\delta} \int_{0}^{L} \varepsilon_{i}\left(u_{\varepsilon_{i}}\right)^{2} d x d t\right\}^{\frac{1}{2}}\left\{\int_{T_{j}+\delta}^{T_{j+1}-\delta} \int_{0}^{L} \frac{W\left(u_{\varepsilon_{i}}\right)}{\varepsilon_{i}} d x d t\right\}^{\frac{1}{2}} .
\end{aligned}
$$

We use the action functional to estimate the first bracket of the RHS of (5.54) and control the second bracket of (5.54) by the energy. For the first bracket, we obtain

$$
\begin{aligned}
& \int_{T_{j}+\delta}^{T_{j+1}-\delta} \int_{0}^{L} \varepsilon_{i}\left(u_{\varepsilon_{i}}\right)^{2} d x d t \\
& =\left.4 S_{\varepsilon_{i}}^{\theta}\left(u_{\varepsilon_{i}}\right)\right|_{t=T_{j}+\delta} ^{T_{j+1}-\delta}-2 \int_{T_{j}+\delta}^{T_{j+1}-\delta} \int_{0}^{L} u_{\varepsilon_{i} t} F_{\varepsilon_{i}}^{\theta} d x d t-\int_{T_{j}+\delta}^{T_{j+1}-\delta} \int_{0}^{L} \frac{\left(F_{\varepsilon_{i}}^{\theta}\right)^{2}}{\varepsilon_{i}} d x d t \\
& =\left.4 S_{\varepsilon_{i}}^{\theta}\left(u_{\varepsilon_{i}}\right)\right|_{t=T_{j}+\delta} ^{T_{j+1}-\delta}-(\mathrm{I})-(\mathrm{II}) .
\end{aligned}
$$

Let the second term of (5.55) be (I) and the third term be (II). We can estimate (I) by the energy. By integrating by parts,

$$
\begin{aligned}
(\mathrm{I}) & =2 \int_{T_{j}+\delta}^{T_{j+1}-\delta} \int_{0}^{L} u_{\varepsilon_{i}}\left(-\varepsilon_{i} u_{\varepsilon_{i} x x}+\frac{W_{\varepsilon_{i}}^{\prime}\left(u_{\varepsilon_{i}}\right)}{\varepsilon_{i}}\right) d x d t \\
& =2 \int_{T_{j}+\delta}^{T_{j+1}-\delta} \int_{0}^{L} \frac{\partial}{\partial t}\left(\frac{\varepsilon_{i}\left(u_{\varepsilon_{i} x}\right)^{2}}{2}+\frac{W_{\varepsilon_{i}}\left(u_{\varepsilon_{i}}\right)}{\varepsilon_{i}}\right) d x d t \\
& =2\left\{\tilde{\mu}_{\varepsilon_{i}}^{T_{j+1}-\delta}([0, L])-\widetilde{\mu}_{\varepsilon_{i}}^{T_{j}+\delta}([0, L])\right\}
\end{aligned}
$$

Since $\mu^{t}([0, L])$ is a constant for $t \in\left(T_{j}, T_{j+1}\right)$, by letting $\varepsilon_{i}$ tend to 0 in (5.56) we obtain

$$
\lim _{i \rightarrow \infty}(\mathrm{I})=2\left\{\eta^{T_{j+1}-\delta}([0, L])-\eta^{T_{j}+\delta}([0, L])\right\}=2 \theta \Delta m_{j}^{\delta} .
$$

Next we consider (II). For fixed $t \in\left(T_{j}+\delta, T_{j+1}-\delta\right)$

$$
\begin{aligned}
\int_{0}^{L} F_{\varepsilon_{i}}^{\theta} u_{\varepsilon_{i} x} d x & =\int_{0}^{L} \frac{\partial}{\partial x}\left(-\frac{\varepsilon_{i}\left(u_{\varepsilon_{i} x}\right)^{2}}{2}+\frac{W_{\varepsilon_{i}}\left(u_{\varepsilon_{i}}\right)}{\varepsilon_{i}}\right) d x \\
& =-\frac{N}{\varepsilon}\left(W_{\varepsilon_{i}}\left(\beta_{\varepsilon_{i}}\right)-W_{\varepsilon_{i}}\left(\alpha_{\varepsilon_{i}}\right)\right) .
\end{aligned}
$$


By the definition of $L^{2}$-norm,

$$
\int_{0}^{L} \frac{\left(F_{\varepsilon_{i}}^{\theta}\right)^{2}}{\varepsilon_{i}} d x \geq \frac{\left(\int_{0}^{L} F_{\varepsilon_{i}}^{\theta} u_{\varepsilon_{i} x} d x\right)^{2}}{\int_{0}^{L} \varepsilon_{i}\left(u_{\varepsilon_{i} x}\right)^{2} d x} .
$$

We set $\Delta T_{j}=T_{j+1}-T_{j}$. Since $\mu^{t}([0, L])$ is a constant for $t \in\left(T_{j}, T_{j+1}\right)$, by (5.42) we obtain

$$
\begin{aligned}
\lim _{i \rightarrow \infty}(\mathrm{II}) & \geq \int_{T_{j}+\delta}^{T_{j+1}-\delta} \liminf _{i \rightarrow \infty} \frac{\left\{-\frac{N}{\varepsilon_{i}}\left(W_{\varepsilon_{i}}\left(\beta_{\varepsilon_{i}}\right)-W_{\varepsilon_{i}}\left(\alpha_{\varepsilon_{i}}\right)\right)\right\}^{2}}{\int_{0}^{L} \varepsilon_{i}\left(u_{\varepsilon_{i} x}\right)^{2} d x} d t \\
& \geq \frac{(-2 \theta N)^{2}}{c_{0} N}\left(\Delta T_{j}-2 \delta\right)=\frac{4 \theta^{2} N}{c_{0}}\left(\Delta T_{j}-2 \delta\right) .
\end{aligned}
$$

By (5.55), (5.57) and (5.60), we obtain

$$
\begin{aligned}
& \lim _{i \rightarrow \infty} \int_{T_{j}+\delta}^{T_{j+1}-\delta} \int_{0}^{L} \varepsilon_{i}\left(u_{\varepsilon_{i}}\right)^{2} d x d t \\
& =\left.4 \lim _{i \rightarrow \infty} S_{\varepsilon_{i}}^{\theta}\left(u_{\varepsilon_{i}}\right)\right|_{T_{j}+\delta} ^{T_{j+1}-\delta}-2 \theta \Delta m_{j}^{\delta}-\frac{4 \theta^{2} N}{c_{0}}\left(\Delta T_{j}-2 \delta\right) .
\end{aligned}
$$

For the second bracket of (5.54), by (5.42), we obtain

$$
\begin{aligned}
\lim _{i \rightarrow \infty} \int_{T_{j}+\delta}^{T_{j+1}-\delta} \int_{0}^{L} \frac{W_{\varepsilon_{i}}\left(u_{\varepsilon_{i}}\right)}{\varepsilon_{i}} d x d t & =\frac{1}{2} \mu^{T_{j}+\delta}([0, L])\left(\Delta T_{j}-2 \delta\right) \\
& \leq \frac{1}{2} c_{0} N \Delta T_{j} .
\end{aligned}
$$

Together with (5.54), (5.61) and (5.62),

$$
\begin{aligned}
\frac{2}{3} \Delta m_{j}^{\delta} & =\lim _{i \rightarrow \infty} I_{\left[T_{j}+\delta, T_{j+1}-\delta\right]}\left(u_{\varepsilon_{i}}\right) \\
& \leq \lim _{i \rightarrow \infty} 2\left(\int_{T_{j}+\delta}^{T_{j+1}-\delta} \int_{0}^{L} \varepsilon_{i}\left(u_{\varepsilon_{i}}\right)^{2} d x d t\right)^{\frac{1}{2}}\left(\int_{T_{j}+\delta}^{T_{j+1}-\delta} \int_{0}^{L} \frac{W\left(u_{\varepsilon_{i}}\right)}{\varepsilon_{i}} d x d t\right)^{\frac{1}{2}} \\
& \leq 2\left\{\left.4 S^{\theta}\right|_{t=T_{j}+\delta} ^{T_{j+1}-\delta}-2 \theta \Delta m_{j}^{\delta}-\frac{4 \theta^{2} N}{c_{0}} \Delta T_{j}-2 \delta\right\}^{\frac{1}{2}}\left\{\frac{c_{0} N}{2} \Delta T_{j}\right\}^{\frac{1}{2}} .
\end{aligned}
$$

Here by letting $\delta$ tend to 0 we obtain

$$
\frac{2}{3} \Delta m_{j}^{0} \leq 2\left\{\left.4 S^{\theta}\right|_{t=T_{j}} ^{T_{j+1}}-2 \theta \Delta m_{j}^{0}-\frac{4 \theta^{2} N}{c_{0}} \Delta T_{j}\right\}^{\frac{1}{2}}\left\{\frac{c_{0} N}{2} \Delta T_{j}\right\}^{\frac{1}{2}} .
$$

Taking the square of the both sides of (5.64),

$$
\left.S^{\theta}\right|_{t=T_{j}} ^{T_{j+1}} \geq \frac{1}{18 c_{0}} \frac{\left(\Delta m_{j}^{0}\right)^{2}}{N \Delta T_{j}}+\frac{\theta}{2} \Delta m_{j}^{0}+\frac{\theta^{2} N}{c_{0}} \Delta T_{j} .
$$


Thus by taking sum with respect to $j$, we obtain

$$
\begin{aligned}
S_{\text {prop. }}^{\theta} & =\left.\sum_{j=1}^{M-1} S^{\theta}\right|_{t=T_{j}} ^{T_{j+1}} \\
& \geq \frac{1}{18 c_{0} N} \sum_{j=1}^{M-1} \frac{\left(\Delta m_{j}^{0}\right)^{2}}{\Delta T_{j}}+\frac{\theta}{2} \sum_{j=1}^{M-1} \Delta m_{j}^{0}+\frac{\theta^{2} N}{c_{0}} \sum_{j=1}^{M-1} \Delta T_{j} .
\end{aligned}
$$

By Jensen's inequality and (5.53),

$$
\sum_{j=1}^{M-1} \frac{\left(\Delta m_{j}^{0}\right)^{2}}{\Delta T_{j}} \geq \frac{\left(\sum_{j=1}^{M-1} \Delta m_{j}^{0}\right)^{2}}{\sum_{j=1}^{M-1} \Delta T_{j}}=\frac{4 L^{2}}{T} .
$$

By (5.53) and (5.67), we obtain

$$
S_{\text {prop. }}^{\theta} \geq \frac{2}{9 c_{0}} \frac{L^{2}}{N T}+\theta L+\frac{\theta^{2} N T}{c_{0}} .
$$

Since $c_{0}=\frac{2 \sqrt{2}}{3}$, the constant is $\frac{2}{9 c_{0}}=\frac{c_{0}}{4}$. Thus we obtain

$$
S_{\text {prop. }}^{\theta} \geq \frac{c_{0} L^{2}}{4 N T}+\theta L+\frac{\theta^{2} N T}{c_{0}}
$$

Consequently Theorem 5.1 follows from (5.49) and (5.69).

Acknowledgement : We would like to thank Professor Yoshihiro Tonegawa for stimulating disucussions and Professor Maria G. Westdickenberg for insightful suggestions and comments. The author was partially supported by Progetto Mecenas, Università di Napoli Federico II and Compagnia di S. Paolo.

\section{References}

[1] Aronson D.G., Weinberger H.F., Multidimensional nonlinear diffusion arising in population genetics, Adv. in Math. 30, (1978), 33-76.

[2] Barles G., Soner H.M., Souganidis P.E., Front propagation and phase field theory, SIAM J. Control and optimization 31 (1993), no. 2, 439-469.

[3] Chen X., Generation and propagation of interfaces for reaction-diffusion equations, J. Differential Equations 96 (1992), 116-141.

[4] Du Q., Liu C., Wang X., A phase field approach in the numerical study of the elastic bending energy for vesicle membranes, J. Commputational Physics 198 (2004), 450-468. 
[5] E W., Ren W., Vanden-Eijnden E., Minimum action method for the study of rare events, Comm. Pure Appl. Math. 57 (2004), no. 5, 637-656.

[6] Faris W.G., Jona-Lasinio G., Large fluctuations for a nonlinear heat equation with noise, J. Phys. A: Math. Gen. 15 (1982), 3025-3055.

[7] Freidlin M. I., Wentzell, A. D., Random perturbations of dynamical systems, (English summary) Second edition. Springer-Verlag, New York, (1998).

[8] Kohn R.V., Otto F., Reznikoff M.G., Vanden-Eijinden E., Action minimizing and sharpinterface limits for the stochastic Allen-Cahn equation, Comm. Pure Appl. Math. 60 (2007), 393-438.

[9] Kohn R.V., Reznikoff M.G., Tonegawa Y., Sharp-interface limit of the Allen-Cahn action functional in one space dimension, Calc. Var. PDEs 25 (2006), no.4, 503-534.

[10] Modica L., The gradient theory of phase transitions and the minimal interface criterion, Arch. Rational Mech. Anal., 98 (2) (1987), 123-142.

[11] Modica L., Mortola S, Il limite nella $\Gamma$-convergenza di una famiglia di funzionali ellittici, (Italian) Boll. Un. Mat. Ital. A (5) 14 (1977), no. 3, 526-529.

[12] Mugnai L., Röger M., The Allen-Cahn action functional in higher dimensions, Interfaces Free Bound. 10 (2008), no. 1, 45-78.

[13] Nagase Y., Tonegawa Y., A singular perturbation problem with integral curvature bound, Hiroshima Math. J. 37 (2007), no. 3, 455-489.

[14] Sternberg P., The effect of a singular perturbation on nonconvex variational problems, Arch. Rational Mech. Anal. 101 (1988), no. 3, 209-260.

[15] Röger M., Schätzle R., On a modified conjecture of De Giorgi, Math. Z. 254 (2006), no. $4,675-714$.

[16] Westdickenberg M. G., Rare events, action minimization, and sharp interface limits, Singularities in PDE and the calculus of variations, 217-231.

[17] Westdickenberg M.G., Tonegawa Y., Higher multiplicity in the one-dimensional AllenCahn action functional, Indiana Univ. Math. J. 56 (2007), no. 6, 2935-2989.

[18] Vanden-Eijnden, E., Westdickenberg M. G., Rare events in stochastic partial differential equations on large spatial domains, J. Stat. Phys. 131 (2008), no. 6, 1023-1038. 